\title{
Lack of quenching for the resonant transmission through an inhomogeneously oscillating quantum well
}

\author{
Qing-feng Sun \\ Department of Physics, Peking University, Beijing 100871, China \\ Jian Wang \\ Department of Physics, The University of Hong Kong, Pokfulam Road, Hong Kong, China \\ Tsung-han Lin \\ CCAST (World Laboratory), P.O. Box 8730, Beijing 100080, China; \\ Institute of Theoretical Physics, Academia Sinica, Beijing 100080, China; \\ and Department of Physics, Peking University, Beijing 100871, China* \\ (Received 1 December 1997; revised manuscript received 17 February 1998)
}

\begin{abstract}
The spectral weights of the wave-function sidebands for a quantum well in the presence of an inhomogeneous electromagnetic (EM) field are studied by introducing a wave function with the form of a Floquet state and then solving the time-dependent Schrödinger equation approximately. The two cases of radiation direction of the EM field parallel and perpendicular to the well axis are considered. We find that the inhomogeneity of the EM field may eliminate the sideband quenching. Based on the spectral weight, the transmission probability through the well is investigated. The energy-level splitting for a special case, the averaged vector potential equal to zero, is also studied. [S0163-1829(98)04128-9]
\end{abstract}

The electron tunneling through nanostructures in the presence of a time-dependent electromagnetic (EM) field has been a subject of increasing interest in the past few years. A number of new effects have been observed, such as the photon-assisted tunneling, ${ }^{1-3}$ the splitting of the Coulomb oscillation peaks, ${ }^{1-3}$ the photon-electron pumping, ${ }^{4,5}$ etc.

Since the pioneering work of Tien and Gordon, ${ }^{6}$ it has been well known that an oscillating potential with frequency $\omega$ can change the energy of an electron state $E$ into a set of energies $E \pm n \hbar \omega(n=0,1,2, \ldots)$, the so-called sideband energies. All phenomena mentioned above are related to the spectral weights of the sidebands. Theoretically, there are two main approaches to study the sideband effects: one is to take the adiabatic approximation and use the Green'sfunction technique, ${ }^{7-12}$ and the other is to solve the timedependent Schrödinger equation directly. ${ }^{13,14}$ In the adiabatic approximation approach, one assumes that the external potential, say, $e V \cos \omega t$, only causes the single-electron energy $\epsilon_{k}$ a rigid shift $\left[\epsilon_{k} \rightarrow \epsilon_{k}(t)=\epsilon_{k}+e V \cos \omega t\right]$, but no transition between different electronic states takes place. ${ }^{7,10-12}$ Then the obtained spectral weight of the $n$th sideband $s_{n}$, which is proportional to $J_{n}^{2}(\alpha)$ (where $\alpha$ $=e V / \hbar \omega$ is a dimensionless variable for the effective field strength), ${ }^{7}$ will vanish at certain values of $\alpha$, corresponding to the zeros of the Bessel function $J_{n}$. This result is usually called sideband quenching. ${ }^{7}$ By using the Schrödinger equation approach, sideband quenching has also been obtained. $^{7,13,14}$

Since the spectral weights are related to the transmission probabilities, one can check the sideband effect by examining the strengths of the side peak around the central resonance, or by measuring the heights of the side step of the $I-V$ curves. Surprisingly, in the experiments by Drexler et al. ${ }^{1}$ and Blick et al.,$^{2}$ no sideband quenching is observed. It is important to notice that almost all the theoretical works that led to sideband quenching always considered the regionally homogeneous fields (either in the quantum-well region or in the lead region). On the other hand, in experiments by Drexler et al. ${ }^{1}$ and Blick et al. ${ }^{2}$ the broadband bowtie antenna is used to couple the microwave fields to the system, which may produce an inhomogeneous field in the quantum-well region. We expect that the inhomogeneity may play an essential role in eliminating sideband quenching.

In order to check this idea, we consider a quantum well applied by a time-dependent field, propagating head-to-head along the $z$ axis, and forming a spatial inhomogeneously standing wave in the quantum-well region. Two special cases, the radiation direction parallel and perpendicular to the well axis, have been investigated (see Fig. 1). Taking the Coulomb gauge, the electric field $\mathbf{E}=-(1 / c)(\partial \mathbf{A} / \partial t)$. By solving the time-dependent Schrödinger equation, the spectral weight of sidebands $s_{l}$ and the transmission probability $T(\epsilon)$ are obtained. It turns out that lack of sideband quenching is found for these inhomogeneous field cases where both
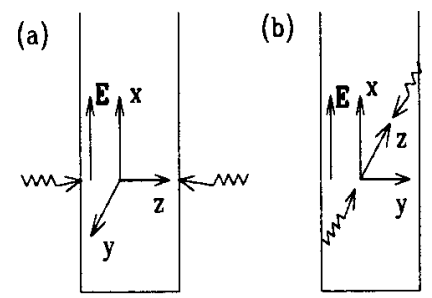

FIG. 1. Schematic description of the model system. $\mathbf{E}$ is the external electric field. (a) and (b) correspond to the cases of the radiation direction parallel and perpendicular to the well axis, respectively. 
$\mathbf{E}(\mathbf{r}, t)$ and $\mathbf{A}(\mathbf{r}, t)$ depend on the coordinates $\mathbf{r}$. Besides, a slight asymmetry of the spectral weights of the sidebands is also found, the same as the result by Wagner. ${ }^{14}$

\section{The case with the radiation direction parallel to well axis}

In order to calculate the spectral weights of the sidebands, we consider that the system is completely confined in the $z$ direction by assuming that the heights of the barriers are infinite, i.e., the confining potential is

$$
V(\mathbf{r})= \begin{cases}+\infty, & |z|>a / 2 \\ 0, & |z| \leqslant a / 2 .\end{cases}
$$

For the realistic system with finite barrier height, the tunneling effect can be described by introducing the phenomenological parameter $\Gamma$, which measures the decay width of the resonant state. To characterize the inhomogeneity of the external field in the well region, we assume that the electric field $\mathbf{E}$ is parallel to the $x$ axis and choose $\mathbf{A}=\left(A_{x}, 0,0\right)$ where $A_{x}$ has the form of $A_{x}=\left(E_{0} c / \omega\right)[g(z)] \cos \omega t$. Expanding $A_{x}$ into Fourier components, one has $A_{x}(z)$ $=\sum_{n} A_{x}\left(q_{n}\right) \exp \left\{i q_{n} z\right\}$, where

$$
A_{x}\left(q_{n}\right)=\frac{E_{0} c}{a \omega} \int_{-a / 2}^{a / 2} g(z) e^{-i q_{n} z} d z \equiv \frac{E_{0} c}{\omega} g_{n},
$$

and $q_{n}=2 \pi n / a(n=0, \pm 1, \pm 2, \ldots)$. Neglecting the higherorder terms of $A_{x}\left(q_{n}\right)$ as in Refs. 6 and 15, the Hamiltonian of the system under consideration can be expressed as

$$
\begin{gathered}
H=H_{0}^{\prime}-\frac{i \hbar e}{m c} A_{x}\left(q_{0}\right) \cos \omega t \frac{d}{d x}, \\
H_{0}^{\prime}=H_{0}-\frac{i \hbar e}{m c} \sum_{n \neq 0} A_{x}\left(q_{n}\right) e^{i q_{n} z} \cos \omega t \frac{d}{d x},
\end{gathered}
$$

where $H_{0}$ is the Hamiltonian without the external field, and the wave function of $H_{0}$ is

$$
f(\mathbf{r}, t)=e^{i\left(k_{x} x+k_{y} y\right)} \cos k_{z} z e^{-i \epsilon_{0} t / \hbar},
$$

with $\epsilon_{0}=\hbar^{2}\left(k_{x}^{2}+k_{y}^{2}+k_{z}^{2}\right) / 2 m$, and $k_{z}=(2 n+1) \pi / a$.

Next, we need to find the solution of the Hamiltonian $H_{0}^{\prime}$. The point here is to find a solution with a special form, the form of the Floquet state. The Floquet state is the analog to a Bloch state when replacing a spatially periodic potential with a time periodic potential. ${ }^{14}$ Therefore, the wave function of the Hamiltonian $H_{0}^{\prime}, \varphi(\mathbf{r}, t)$, should be expressed as

$$
\varphi(\mathbf{r}, t)=e^{-i \epsilon t / \hbar} u(\mathbf{r}, t)=e^{-i \epsilon t / \hbar} \sum_{l} u_{l}(\mathbf{r}) e^{i l \omega t}
$$

$u_{l}(\mathbf{r})$ can be expanded into the series of $A_{x}\left(q_{n}\right)$ :

$$
u_{l}(\mathbf{r})=u_{l}^{(0)}(\mathbf{r})+\sum_{n \neq 0} u_{l, q_{n}}^{(1)}(\mathbf{r}) A_{x}\left(q_{n}\right)+\cdots
$$

Notice that $\varphi(\mathbf{r}, t)$ should return to $f(\mathbf{r}, t)$ if $A_{x}\left(q_{n}\right)=0$; so only one term in the set of $\left\{u_{l}^{(0)}(\mathbf{r})\right\}$ is not zero, which is denoted by $u_{0}^{(0)}(\mathbf{r})$. In fact, which one we choose as the nonzero term does not affect the result.
Substituting the expression of $\varphi(\mathbf{r}, t)$ into the timedependent Schrödinger equation of $H_{0}^{\prime}$ Eq. (3), only keeping the terms with the first order of $A_{x}\left(q_{n}\right)$, and by comparing the coefficient of the terms with the same $\exp \{i l \omega t\}$, one easily finds

$$
\begin{aligned}
& (l=0) \quad H_{0} u_{0}(\mathbf{r})=\epsilon u_{0}(\mathbf{r}), \\
& (l=1) \quad H_{0} u_{1}(\mathbf{r})-\frac{i \hbar e}{2 m c} \sum_{n \neq 0} A_{x}\left(q_{n}\right) e^{i q_{n} z} \frac{d}{d x} u_{0}(\mathbf{r}) \\
& =(\epsilon-\hbar \omega) u_{1}(\mathbf{r}), \\
& (l=-1) \quad H_{0} u_{-1}(\mathbf{r})-\frac{i \hbar e}{2 m c} \sum_{n \neq 0} A_{x}\left(q_{n}\right) e^{i q_{n} z} \frac{d}{d x} u_{0}(\mathbf{r}) \\
& =(\epsilon+\hbar \omega) u_{-1}(\mathbf{r}), \\
& (l \neq 0, \pm 1) \quad H_{0} u_{l}(\mathbf{r})=(\epsilon-l \hbar \omega) u_{l}(\mathbf{r}) .
\end{aligned}
$$

Notice that the boundary condition $\left.\varphi(\mathbf{r}, t)\right|_{z= \pm a / 2}=0$ corresponds to $\left.u_{l}(\mathbf{r})\right|_{z= \pm a / 2}=0$.

From Eq. (7), which is an eigenequation, one easily obtains $u_{0}(\mathbf{r})=e^{i k_{x} x} e^{i k_{y} y} \cos k_{z} z$, and $\epsilon=\hbar^{2}\left(k_{x}^{2}+k_{y}^{2}+k_{z}^{2}\right) / 2 m$ $=\epsilon_{0}$ with $k_{z}=(2 n+1) \pi / a$. It should be pointed out that $\epsilon$ is independent with $A_{x}\left(q_{n}\right)$ in the first-order approximation.

Then we solve Eq. (8). Substituting $u_{0}(\mathbf{r})$ and $u_{1}(\mathbf{r})$, Eq. (6), into Eq. (8), and noticing that $u_{1}^{0}(\mathbf{r})=0$, one easily obtains

$$
\begin{aligned}
H_{0} u_{1, q_{n}}^{(1)}(\mathbf{r}) & +\frac{\hbar k_{x} e}{2 m c} e^{i q_{n} z} \cos k_{z} z e^{i k_{x} x} e^{i k_{y} y} \\
& =\left(\epsilon_{0}-\hbar \omega\right) u_{1, q_{n}}^{(1)}(\mathbf{r}) .
\end{aligned}
$$

Then let $u_{1, q_{n}}^{(1)}(\mathbf{r})=u_{1, q_{n}}^{(1)}(z) e^{i k_{x} x} e^{i k_{y} y}$; one has

$$
\begin{array}{r}
-\frac{\hbar^{2}}{2 m} \frac{d^{2}}{d z^{2}} u_{1, q_{n}}^{(1)}(z)+\frac{\hbar k_{x} e}{2 m c} e^{i q_{n} z} \cos k_{z} z \\
=\left(\epsilon_{0}-\frac{\hbar^{2}\left(k_{x}^{2}+k_{y}^{2}\right)}{2 m}-\hbar \omega\right) u_{1, q_{n}}^{(1)}(z) .
\end{array}
$$

Noticing that the above differential equation is not an eigenequation, and the general solution is

$$
\begin{aligned}
u_{1, q_{n}}^{(1)}(z)= & C_{q_{n}} e^{i k_{z}^{\prime} z}+D_{q_{n}} e^{-i k_{z}^{\prime} z} \\
& -\frac{\hbar k_{x} e}{4 m c}\left[\frac{e^{i\left(q_{n}+k_{z}\right) z}}{\epsilon_{q_{n}+k_{z}}-\epsilon_{0}+\hbar \omega}+\frac{e^{i\left(q_{n}-k_{z}\right) z}}{\epsilon_{q_{n}-k_{z}}-\epsilon_{0}+\hbar \omega}\right] \\
\equiv & C_{q_{n}} e^{i k_{z}^{\prime} z}+D_{q_{n}} e^{-i k_{z}^{\prime} z}+f_{q_{n}}(z),
\end{aligned}
$$

where $\quad k_{z}^{\prime}=\sqrt{k_{z}^{2}-2 m \omega / \hbar}, \quad \epsilon_{q_{n} \pm k_{z}}=\hbar^{2}\left[k_{x}^{2}+k_{y}^{2}+\left(q_{n}\right.\right.$ $\left.\left.\pm k_{z}\right)^{2}\right] / 2 m$, and $f_{q_{n}}(z)$ is a compact notation of the last term on the right-hand side of $u_{1, q_{n}}^{(1)}(z)$, and $C_{q_{n}}$ and $D_{q_{n}}$ are arbitrary constant that should be determined by the boundary condition, $u_{1, q_{n}}^{(1)}( \pm a / 2)=0$. From the boundary condition, one has the following equations: 


$$
\begin{gathered}
C_{q_{n}} e^{i k_{z}^{\prime} a / 2}+D_{q_{n}} e^{-i k_{z}^{\prime} a / 2}+f_{q_{n}}(a / 2)=0, \\
C_{q_{n}} e^{-i k_{z}^{\prime} a / 2}+D_{q_{n}} e^{i k_{z}^{\prime} a / 2}+f_{q_{n}}(-a / 2)=0 .
\end{gathered}
$$

Then the coefficients $C_{q_{n}}$ and $D_{q_{n}}$ are determined, and $u_{1}(\mathbf{r})$ is obtained immediately. Notice that $f_{q_{n}}(-a / 2)=f_{q_{-n}}(a / 2)$ $=-f_{q_{n}}(a / 2)$, one easily finds $C_{q_{-n}}+C_{q_{n}}=0$ and $D_{q_{n}}$ $+D_{q_{-n}}=0$. If one only considers the case of $A_{x}\left(q_{n}\right)$ $=A_{x}\left(q_{-n}\right)\left[\right.$ i.e., $\left.A_{x}(z)=A_{x}(-z)\right], u_{l}(\mathbf{r})$ will reduce to

$$
u_{1}(\mathbf{r})=\sum_{n \neq 0} \frac{-\hbar k_{x} e}{2 m c} \frac{\cos \left(k_{z}+q_{n}\right) z}{\epsilon_{q_{n}+k_{z}}-\epsilon_{0}+\hbar \omega} e^{i k_{x} x} e^{i k_{y} y} A_{x}\left(q_{n}\right) .
$$

By using the same procedure, we can solve Eqs. (9) and (10) as

$$
\begin{gathered}
u_{-1}(\mathbf{r})=\sum_{n \neq 0} \frac{-\hbar k_{x} e}{2 m c} \frac{\cos \left(k_{z}-q_{n}\right) z}{\epsilon_{q_{n}-k_{z}}-\epsilon_{0}+\hbar \omega} e^{i k_{x} x} e^{i k_{y} y} A_{x}\left(q_{n}\right) \\
u_{l}(\mathbf{r})=0 \quad(l \neq 0, \pm 1)
\end{gathered}
$$

Then substituting $u_{l}(\mathbf{r})$ into Eq. (5), and the wave function of the Hamiltonian $H_{0}^{\prime}, \varphi(\mathbf{r}, t)$, can be obtained as

$$
\begin{aligned}
\varphi(\mathbf{r}, t)= & e^{i\left(k_{x} x+k_{y} y\right)} e^{-i \epsilon_{0} t / \hbar}\left\{\cos k_{z} z-\frac{\hbar e k_{x}}{2 m c}\right. \\
& \times \sum_{n \neq 0}\left[e^{i \omega t} \frac{\cos \left(q_{n}+k_{z}\right) z}{\epsilon_{q_{n}+k_{z}}-\epsilon_{0}+\hbar \omega}\right. \\
& \left.\left.+e^{-i \omega t} \frac{\cos \left(q_{n}-k_{z}\right) z}{\epsilon_{q_{n}-k_{z}}-\epsilon_{0}-\hbar \omega}\right] A_{x}\left(q_{n}\right)\right\}
\end{aligned}
$$

Referring to the Tien-Gordon theory, ${ }^{6}$ we finally find the wave function of the total Hamiltonian $H$,

$$
\psi(\mathbf{r}, t)=\varphi(\mathbf{r}, t) \sum_{l} J_{l}(\alpha) e^{-i l \omega t},
$$

where

$$
\alpha=\frac{e k_{x} A_{x}\left(q_{0}\right)}{m c \omega}=\frac{E_{0} e k_{x} q_{0}}{a m \omega^{2}}
$$

is a dimensionless variable for the effective field strength, and $\psi(\mathbf{r}, t)$ can be expressed in the following form:

$$
\psi(\mathbf{r}, t)=\sum_{l} \sum_{\tilde{k}_{z}} B_{l}\left(\tilde{k}_{z}\right) e^{i\left(k_{x} x+k_{y} y\right)} \cos \tilde{k}_{z} z e^{-i\left(\epsilon_{0}+l \hbar \omega\right) t / \hbar},
$$

where $\tilde{k}_{z}=(2 n+1) \pi / a$. Since the set of the functions $\cos \left(\tilde{k}_{z} z\right)$ with different $\tilde{k}_{z}$ is orthonormal, the spectral weight of the sidebands can be expressed as $S_{l}=\Sigma_{\tilde{k}_{z}}\left|B_{l}\left(\tilde{k}_{z}\right)\right|^{2}$. If only the ground state $\left(k_{z}=\pi / a\right)$ of the electron in the well is taken into account, then $S_{l}$ is given by

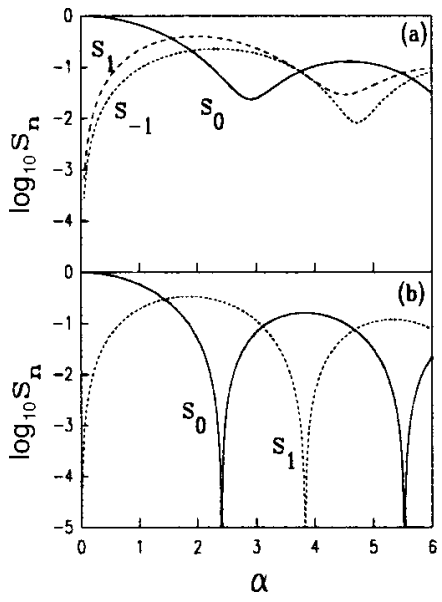

FIG. 2. The spectral weights $s_{l}$ vs $\alpha$ for the case of the radiation direction parallel to the well axis, where $\omega=1, a=0.1, k_{x}=k_{y}$ $=k_{z}=\pi / a$, and in units of $e=\hbar=c=m=1$. (a) $E_{x}$ $=E_{0} \cos q z \sin \omega t$ with $q=\pi / a$; (b) the homogeneous case with $E_{x}=E_{0} \sin \omega t$ for comparison.

$$
\begin{aligned}
S_{l}\left(\epsilon_{0}\right)= & \left|J_{l}(\alpha)+\frac{\hbar e k_{x}}{2 m c}\left[h_{-}(1) J_{l-1}(\alpha)+h_{+}(1) J_{l+1}(\alpha)\right]\right|^{2} \\
& +\left(\frac{\hbar e k_{x}}{2 m c}\right)^{2} \sum_{n>0} \mid h_{+}(n) J_{l+1}(\alpha)+h_{-}(n+1) J_{l-1}(\alpha) \\
& +h_{+}(-n-1) J_{l+1}(\alpha)+\left.h_{-}(-n) J_{l-1}(\alpha)\right|^{2},
\end{aligned}
$$

where

$$
h_{ \pm}(n) \equiv \frac{A_{x}\left(q_{n}\right)}{\epsilon_{q_{n}+k_{z}}-\epsilon_{0} \pm \hbar \omega} .
$$

Let $s_{l}$ denote the normalized spectral weight of the sidebands, $s_{l}=S_{l}\left(\epsilon_{0}\right) / \Sigma_{l} S_{l}\left(\epsilon_{0}\right)$.

The dimensionless variable obtained in this work is

$$
\alpha=\frac{e k_{x} A_{x}\left(q_{0}\right)}{m c \omega}=\frac{E_{0} e k_{x} q_{0}}{a m \omega^{2}},
$$

which is scaled as $\omega^{-2}$, the same as the result obtained by Wagner, ${ }^{14}$ but different from the scaling of $\omega^{-1}$ obtained by the adiabatic approximation approach. Moreover, now $\alpha$ depends on the transverse momentum $k_{x}$. From Eq. (21) one easily sees that when $k_{x}=0, s_{l}(l \neq 0)$ is identically equal to zero for any amplitude of the external field $E_{0}$, which means that the energy level $\epsilon_{0}$ does not split into sidebands.

Now let us consider the more interesting case with $k_{x} \neq 0$. From Eq. (21), the spectral weight of the sidebands $s_{l}$ is highly nonlinear with $E_{0}$. Since the vector potential $\mathbf{A}(\mathbf{r}, t)$ depends on $\mathbf{r}$, the spectral weight of the sidebands $s_{l}$ will be related to the Bessel functions $J_{l-1}(\alpha), J_{l}(\alpha)$, and $J_{l+1}(\alpha)$. It is impossible to find a nonzero value of $\alpha$ making all three Bessel functions $\left[J_{l-1}(\alpha), J_{l}(\alpha)\right.$, and $\left.J_{l+1}(\alpha)\right]$ be zero. Therefore, with increasing the amplitude of the external field $E_{0}$, the spectral weight of the sidebands $s_{l}$ varies, but never vanishes, i.e., the sideband quenching is eliminated under the inhomogeneity of the EM field. This behavior is shown in Fig. 2(a) for $E_{x}=E_{0} \cos q z \sin \omega t$. One can also see a slight asymmetry for the sidebands $s_{ \pm l}$. For compari- 
son, Fig. 2(b) presents the case of a homogeneous field by taking $E_{x}=E_{0}$ sin $\omega t$. In this case one simply has $s_{l}$ $=J_{l}^{2}(\alpha)$, exhibiting the sideband quenching.

\section{The case with the radiation direction perpendicular to the well axis}

In this case the system is confined in the $y$ direction [see Fig. 1(b)], and the confining potential is

$$
V(\mathbf{r})=\left\{\begin{array}{l}
+\infty, \quad|y|>a / 2 \\
0, \quad|y| \leqslant a / 2
\end{array}\right.
$$

Now the vector potential is set to be $\mathbf{A}=\left(A_{x}, 0,0\right)$ with $A_{x}$ $=\left(E_{0} c / \omega\right) g(z) \cos \omega t$. Considering the standing wave as a periodic function, i.e., $A_{x}(z+b)=A_{x}(z)$, and taking the Fourier expansion, one has $A_{x}(z)=\Sigma_{n} A_{x}\left(q_{n}\right) \exp \left(i q_{n} z\right)$ with

$$
A_{x}\left(q_{n}\right)=\frac{E_{0} c}{b \omega} \int_{-b / 2}^{b / 2} g(z) e^{-i q_{n} z} d z \equiv \frac{E_{0} c}{\omega} g_{n},
$$

where $q_{n}=2 \pi n / b(n=0, \pm 1, \ldots)$. By neglecting the higherorder terms of $A_{x}\left(q_{n}\right)$ as in case 1 , the Hamiltonian can be written as the same form as in Eq. (3). Then one obtains the wave function as

$$
\begin{aligned}
\psi(\mathbf{r}, t)= & e^{-i \epsilon_{0} t / \hbar} e^{i\left(k_{x} x+k_{z} z\right)} \cos k_{y} y \\
& \times\left\{1-\frac{\hbar e k_{x}}{2 m c} \sum_{n \neq 0} e^{i q_{n} z}\left[h_{+}(n) e^{i \omega t}+h_{-}(n) e^{-i \omega t}\right]\right\} \\
& \times \sum_{l=-\infty}^{+\infty} J_{l}(\alpha) e^{-i l \omega t}
\end{aligned}
$$

where $k_{y}$ only takes the values of $(2 n+1) \pi / a \quad(n=0$, $\pm 1, \ldots)$. Different from case 1 , there is no restriction of $A_{x}(z)=A_{x}(-z)\left[\right.$ or $\left.A_{x}\left(q_{n}\right)=A_{x}\left(q_{-n}\right)\right]$ in this case. Then the spectral weight of the sideband obtained is

$$
\begin{aligned}
s_{l}\left(\epsilon_{0}\right)= & B\left[J_{l}^{2}(\alpha)+\left(\frac{\hbar e k_{x}}{2 m c}\right)^{2} \sum_{n \neq 0} \mid h_{+}(n) J_{l+1}(\alpha)\right. \\
& \left.+\left.h_{-}(n) J_{l-1}(\alpha)\right|^{2}\right]
\end{aligned}
$$

where $B$ is the normalization factor.

Figure 3(a) shows $s_{l}$ vs $E_{0}$ (or $\alpha$ ). Again, the spectral weight of the sidebands $s_{l}$ has a lack of quenching at any values of $E_{0}$ and exhibits a slight asymmetry (not shown in the figure). These features originated from the inhomogeneity of the electric field in the quantum-well region. We also see from Fig. 3(a) that $s_{l}$ saturates at large $\alpha$. Figure 3(b) presents the spectral weight of the sidebands for the case with homogeneous field for comparison, which clearly shows the sideband quenching and the symmetric spectral weights.

Based on the spectral weight $s_{l}$, one can calculate the electron transmission probability through the quantum well in the presence of the external field $T(\epsilon) .{ }^{14}$ Let $\Gamma^{L}\left(\Gamma^{R}\right)$ denote the half width of the resonant energy level due to the tunneling through the left (right) barrier to the left (right) lead without the external field. Since the states of different

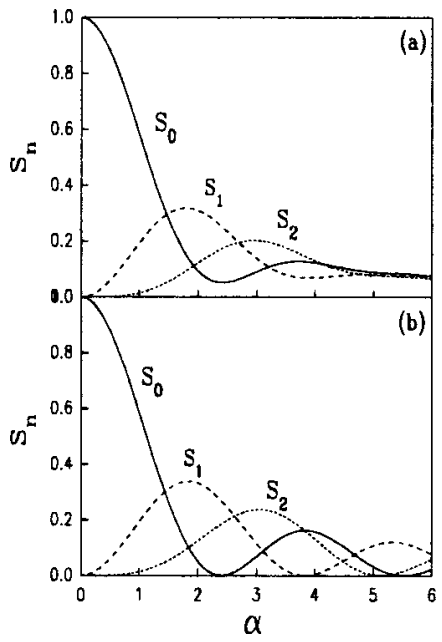

FIG. 3. The spectral weights $s_{l}$ vs $\alpha$ for the case of the radiation direction perpendicular to the well axis, where $\omega=1, a=b=0.5$, and $k_{x}=k_{y}=k_{z}=\pi / a$. (a) $E_{x}=E_{0} \cos q z \sin \omega t$ (for $|z| \leqslant b / 2$ ) with $q=1.5 \pi / a$; (b) the homogeneous case with $E_{x}=E_{0} \sin \omega t$ for comparison.

sidebands are orthonormal, the transmission probability $T(\epsilon)$ can be obtained by the Breit-Wigner formula as

$$
T(\epsilon)=\sum_{l} \frac{s_{l} \Gamma^{L} \Gamma^{R}}{\left(\epsilon-\epsilon_{0}+l \hbar \omega\right)^{2}+(\Gamma / 2)^{2}},
$$

where $\Gamma=\Gamma^{L}+\Gamma^{R}$. Figure 4 shows $T$ vs $\epsilon$ for case 2. In Fig. 4(a), we take $A_{x}\left(q_{0}\right)=E_{0} c / \omega, A_{x}\left(q_{ \pm 1}\right)=0.5 E_{0} c / \omega$, and $A_{x}\left(q_{n}\right)=0$ for all other $q_{n}$ 's. The sideband peaks do not vanish at any $\alpha$ (except $\alpha=0$ ) with a slight asymmetry (too small to see). With the increasing of $E_{0}$ (or $\alpha$ ), more and more sideband peaks emerge. These features are in agreement with the experiments by Drexler et al. ${ }^{1}$ For comparison, Fig. 4(b) presents the result of homogeneous field with $A_{x}\left(q_{n}\right)=0(n \neq 0)$, showing a strong quenching for the main peak at $\alpha=2.4$.

Finally, we study a special case with $A_{x}\left(q_{0}\right)=0$, i.e., the average of vector potential $\mathbf{A}(\mathbf{r}, t)$ on the coordinates $\mathbf{r}$ equal

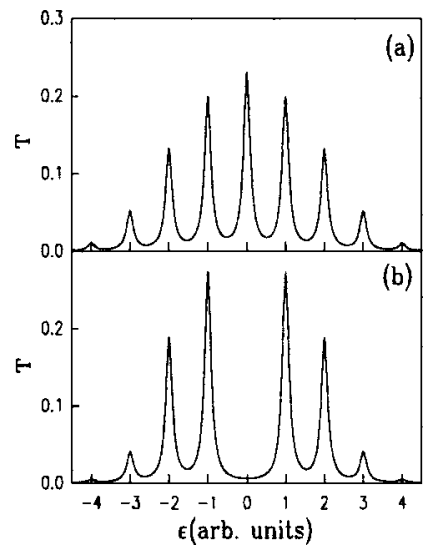

FIG. 4. $T(\epsilon)$ vs $\epsilon$ for the case of the radiation direction perpendicular to the well axis, where $\omega=1, a=b=1, k_{x}=k_{y}=k_{z}=\pi / a$, $\alpha=2.4, \quad$ and $\quad \Gamma^{L}=\Gamma^{R}=0.1 . \quad$ (a) $\quad A_{x}\left(q_{0}\right)=E_{0} c / \omega, \quad A_{x}\left(q_{ \pm 1}\right)$ $=0.5 E_{0} c / \omega$, and all other $A_{x}\left(q_{n}\right)$ are equal to zero; (b) all $A_{x}\left(q_{n}\right)$ are equal to zero except $A_{x}\left(q_{0}\right)=E_{0} c / \omega$. 


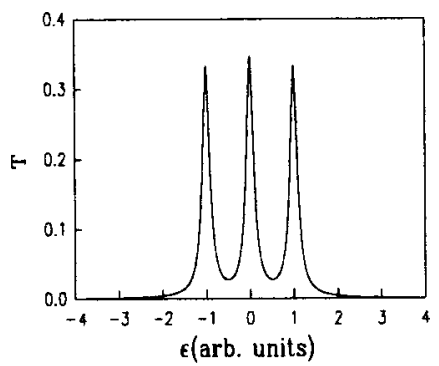

FIG. 5. $T(\epsilon)$ vs $\epsilon$ for the case of the radiation direction perpendicular to the well axis and with $A_{x}\left(q_{0}\right)=0$, where $\omega=1, a=b$ $=0.4, \quad k_{x}=k_{y}=k_{z}=\pi / a, \quad E_{0}=0.1, \quad \Gamma^{L}=\Gamma^{R}=0.1, \quad E_{x}$ $=E_{0} \sin q z \sin \omega t($ at $|z| \leqslant z / 2)$ with $q=1.5 \pi / a$.

to zero, leading to $\alpha=0$. In this particular case the main resonant peak will mainly be splitted into three sideband peaks (see Fig. 5). From Eq. (21) or Eq. (26), one can easily find that only $s_{0}$ and $s_{ \pm 1}$ have significant amplitudes; the others are zero in the first-order approximation. If one considers the second-order terms, $A_{x}^{2}\left(q_{n}\right)$, the sidebands $s_{ \pm 2}$ will emerge, but the ratio $s_{ \pm 2} / s_{ \pm 1}$ is about

*Mailing address.

${ }^{1}$ H. Drexler, J. S. Scott, S. J. Allen, K. L. Campman, and A. C. Gossard, Appl. Phys. Lett. 67, 2816 (1995).

${ }^{2}$ R. H. Blick, R. J. Haug, D. W. van der Weide, K. von Klitzing, and K. Eberl, Appl. Phys. Lett. 67, 3924 (1995).

${ }^{3}$ T. H. Oosterkamp, L. P. Kouwenhoven, A. E. A. Koolen, N. C. van der Vaart, and C. J. P. M. Harmans, Phys. Rev. Lett. 78, 1536 (1997).

${ }^{4}$ L. P. Kouwenhoven, S. Jauhar, J. Orenstein, P. L. McEuen, Y. Nagamune, J. Motohisa, and H. Sakaki, Phys. Rev. Lett. 73, 3443 (1994).

${ }^{5}$ L. P. Kouwenhoven, S. Jauhar, K. McCormick, D. Dixon, P. L. McEuen, Yu. V. Nazarov, N. C. van der Vaart, and C. T. Foxon, Phys. Rev. B 50, 2019 (1994).

${ }^{6}$ P. K. Tien and J. P. Gordon, Phys. Rev. 129, 647 (1963). $e A_{x} / c P_{x} \approx e E_{0} / \omega \hbar k_{x} \approx 0.01$ (all parameters are the same as in Fig. 5, and in the units of $e=\hbar=c=1$ ).

In summary, we have studied the sideband effect of the electron transmission through a quantum well in the presence of a time-dependent field. We find that whether the sideband quenching appears is critically dependent on the homogeneity of the field in the quantum-well region. Lack of sideband quenching is found for the inhomogeneous field case, which is in agreement with the experiments by Drexler et al. ${ }^{1}$ and Blick et $a l^{2}$ Recently, we have noticed that Oosterkamp et al. reported the electron tunneling through a quantum dot in which one can see a sideband quenching. ${ }^{3}$ We guess it probably comes from the homogeneity of the field in the dot region. In addition, two characters are predicted in this work: no splitting of the resonant energy level for $k_{x}=0$; and mainly splitted into three sidebands for $A_{x}\left(q_{0}\right)=0$.

This work was partially supported by the National Natural Science Foundation of China and the Doctoral Program Foundation of the Institution of Higher Education. J.W. was supported by a CRCG grant from the University of Hong Kong.
${ }^{7}$ M. Wagner, Phys. Rev. B 49, 16544 (1994); Phys. Rev. A 51, 798 (1995).

${ }^{8}$ R. Aguado and G. Platero, Phys. Rev. B 55, 12860 (1997).

${ }^{9}$ Y. Goldin and Y. Avishai, Phys. Rev. B 55, 16359 (1997).

${ }^{10}$ C. A. Stafford and Ned S. Wingreen, Phys. Rev. Lett. 76, 1916 (1996).

${ }^{11}$ Antti-Pekka Jauho, N. S. Wingreen, and Y. Meir, Phys. Rev. B 50, 5528 (1994).

${ }^{12}$ Qing-feng Sun and Tsung-han Lin, J. Phys.: Condens. Matter 9, 4875 (1997); Phys. Rev. B 56, 3591 (1997).

${ }^{13}$ D. Sokolovski, Phys. Rev. B 37, 4201 (1988).

${ }^{14}$ M. Wagner, Phys. Rev. Lett. 76, 4010 (1996).

${ }^{15}$ J. Inarrea, G. Platero, and C. Tejedor, Phys. Rev. B 50, 4581 (1994). 